\title{
Investigation of Structure-Function Relationship of Long-Distance Transport in Plants: New Imaging Tools to Answer Old Questions
}

\author{
Daniel L Mullendore, Daniel R Froelich, Sierra Beecher, Tim J Ross-Elliott, Jan Knoblauch, Michael \\ Knoblauch
}

Plant Cell Biology Lab, School of Biological Sciences, Washington State University, Pullman WA

The phloem is a plant tissue that connects distant plant parts via the sieve tube system. It is a major player to maintain organismal integrity due to its activity in translocation of photoassimilates and distribution of long distance signals [1, 2]. Almost all food consumed by humans, or biomass produced for bioenergy, has at one point been translocated through the phloem. While major research efforts focus on optimization of photosynthesis to tackle biomass and food production, phloem loading and transport is poorly understood. Photosynthetic efficiency is, however, dependent on phloem transport and is down-regulated if production exceeds export significantly. Therefore, a better understanding of processes dictating long distance transport is essential.

One of the major reasons for our poor understanding of phloem structure-function relations is that preparation induces immediate artefacts $[3,4]$. The preparation for electron optic observation requires small sample sizes. While cutting tissue samples before fixation has only minor effects on standard cell types such as parenchyma-, mesophyll- or epidermis cells, sample preparation of sieve elements usually leads to massive artifact generation due to the high conductivity combined with high turgor in sieve tubes. Cutting the tube system results in an instant pressure release followed by dislocation and structural alteration of subcellular components [5]. A large group of investigators tackled this problem from the 1960's to the 1980's. Hundreds of publications later, the results were still confusing [1].

One of the major problems was that investigators had no in vivo reference. While electron microscopy provides unprecedented resolution, samples often contain severe preparation and dehydration induced artefacts. Without a (low resolution) reference of the location and structure of components in vivo, it is very difficult to interpret and to optimize preparation protocols. Even though some of the micrographs might have shown nearly natural conditions, it is unclear which ones. Over the last years we have developed a series of methods for in situ studies of sieve tubes by confocal and super resolution microscopy $(6,7,8)$. Based on our observations, we have developed a preparation method to maintain the subcellular structure of sieve tubes close to the natural state.

We developed microscopy rhizosphere chambers (Micro-ROCs) which are a square plant pot with one wall consisting of a \#1.5 coverslip. A micro-pore fabric is attached flat against the coverslip from the inside of the pot. The pot is filled with soil and a seed is placed between the meshwork and the coverslip. The mesh forces the germinating root to grow two dimensionally along the coverslip, while the root hairs are in contact with the soil medium to ensure natural growth conditions. For observation, the whole pot is put under the microscope to ensure minimum disturbance. The plants can be grown for weeks through their entire life cycle.

We investigated the phloem in Arabidopsis roots in situ and were able to study the dynamics of phloem transport and phloem unloading. The generation of transgenic plants expressing fluorescent proteins tagged to different structures and organelles allowed us to study the precise location of the structures [8]. 
Our investigations generated with micro-ROCs revealed structures that had never been described before. To gain more insight by higher resolution, we attempted to generate a preparation protocol for electron microscopy. Since cutting inevitably generates artefacts, we used whole young plants for tissue preparation and embedding. Standard preparation methods failed to preserve structures observed in situ. Therefore we attempted to explore freezing techniques. The phloem is at least 3-4 cell layers deep inside the tissue which calls for high pressure freezing. Unfortunately the sample size did not allow making use of this technology. Therefore we plunge froze whole seedlings in slushy nitrogen followed by freeze substitution. While the depth of the phloem inside the tissue reduces heat transfer rates, the high sugar content reduces water crystal formation. It turned out that neighboring cells like parenchyma and mesophyll cells showed severe freezing artifacts, but sieve elements and their adjacent companion cells were nicely preserved. Most importantly, however, we were able to image structures that had never been described in sieve elements and match perfectly the location and shape of structures found in in situ observations[8].

We now have all the tools necessary to investigate the structure and physiology of phloem transport and close a major gap in our understanding of plant function. Besides phloem studies, micro-ROCs are suitable to study root physiology and root soil communities in situ.

[1] ed HD Behnke, RD Sjolund (1990) Sieve elements (Springer, Berlin, New York)

[2] M Knoblauch, WS Peters, Photosynthesis Research 117 (2013) 189-196

[3] M Knoblauch, KJ Oparka, The Plant Journal 70 (2012) 147-156

[4] M Knoblauch, WS Peters, Plant, Cell, Environment 33 (2010) 1439 - 1452

[5] M Knoblauch, AJE van Bel, The Plant Cell 10 (1998) 35-50

[6] DL Mullendore et al., The Plant Cell 22 (2010) 579-593

[7] DF Froelich et al., The Plant Cell 23 (2011) 4428-4445

[8] Anstead et al., Plant Cell Physiology 53 (2012) 1033-1042

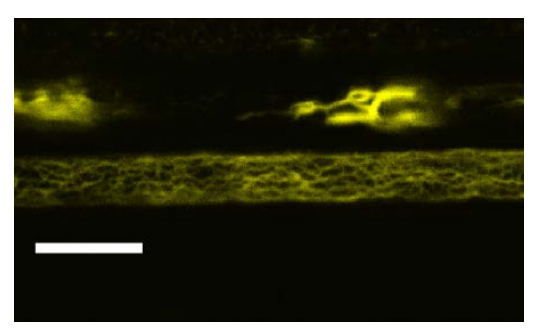

Figure 1. Imaging of P-proteins taggd with YFP in an intact sieve tube of a transgenic Arabidospsis plant. Bar $=10 \mu \mathrm{m}$.

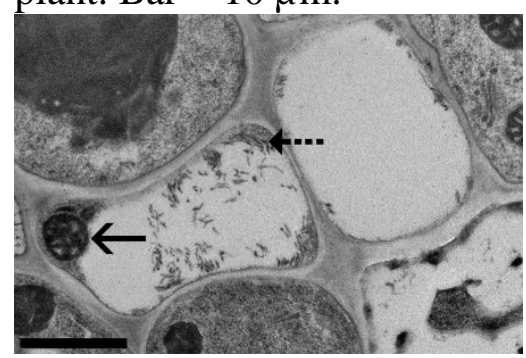

Figure 2. TEM micrograph of Arabidopsis sieve elements after plunge freezing and freeze substitution. The sieve elements contain well preserved mitochondria (solid arrow) and P-protein filaments (dashed arrow). Bar $=1 \mu \mathrm{m}$. 